\title{
A Mathematical Model of the Cell Differentiation in Multicellular Eukaryotes
}

\author{
Jinya Otsuka \\ JO Institute of Biophysics, Higashi-Ohizumi, Nerima-ku, Tokyo, Japan \\ Email: jin.otsuka@kyj.biglobe.ne.jp
}

How to cite this paper: Otsuka, J. (2020) A Mathematical Model of the Cell Differentiation in Multicellular Eukaryotes. Applied Mathematics, 11, 157-171.

https://doi.org/10.4236/am.2020.113014

Received: January 2, 2020

Accepted: March 8, 2020

Published: March 11, 2020

Copyright (c) 2020 by author(s) and Scientific Research Publishing Inc. This work is licensed under the Creative Commons Attribution International License (CC BY 4.0).

http://creativecommons.org/licenses/by/4.0/

\begin{abstract}
The cell differentiation in multicellular eukaryotes is one of the most curious phenomena. The recent gene and genome sequencing reveals that most of differentiated cells in a multicellular eukaryote carry a common genome and that such a genome contains the expanded repertoire of genes of proteins associated with the cell-cell adhesion, intercellular and intracellular signal transduction and transcriptional regulation. The cell differentiation occurs in the assembly consisting of a large number of cells after the cell proliferation, and this process is regarded as a stochastic process. Its formulation starts with the master equation in the present paper. The cell differentiation is reproduced in the equation of the most probable path derived from the master equation, when the short-range and long-range interactions between the cells as well as the transition probability between the proliferation and differentiation modes are considered. Moreover, the equation of the most probable path explains the experimental results such as the "memory", tissue culture and the preparation of induced pluripotent stem (iPS) cells in embryology, if the long-range interaction is considered to be the regulation of gene transcription under the influence of intracellular signal transduction from the receptor accepting the ligand secreted by other types of cells and the short-range interaction is considered to stabilize the intracellular signal transduction by the contact between the same type of cells. The "organizer" found in the initial development of embryo is also explained as the cells that preferentially express the specific gene of a ligand to rouse the long-range interaction. In conclusion, the present study proposes that the complicated intercellular and intracellular signal transduction causing the cell differentiation is ascribed to the long-range interaction between distinctive types of cells and the short-range interaction between the same type of cells.
\end{abstract}

\section{Keywords}

Stochastic Process, Long-Range Interaction, Short-Range Interaction, Signal 
Transduction, Transcriptional Regulation

\section{Introduction}

The descriptive embryology sketches a broad outline of the early stages of development, recognizing two basic phenomena; the arising of differences between the various parts of the living matter and the moulding of a mass of tissues into a coherent structure. Cell differentiation underlies the former phenomenon and the latter phenomenon is called "morphogenesis". Experimental embryology inquired into the causality in such development by cutting and transplanting some parts of embryo to other regions. In this attempt, it is found in newt that the dorsal vegetative quadrant of gastrula is the organization centre or organizer for forming the embryonic axis [1] [2], although any half before gastrulation becomes a complete embryo. The primary organizer not only induces the ectoderm above it to brain and spinal cord but also the organizer itself becomes notochord and metamere. Such induced organs are considered to act as secondary, tertiary, quaternary organizers and so on, acting one after another. However, the time course of such development is only interpreted in terms of ooplasmic segregation, evocation and field action [3]. The phenomenon "morphogenesis" inspires a mathematician to propose a theory of catastrophe on the basis of universal unfolding of the germ of a mathematical function [4], although the mathematical function and variable(s) used in this theory are still not physically founded in the development. On the other hand, the cell differentiation is not inquired but traditionally classified into two extremes; the whole future pattern of a body is delineated by localized determinants in the egg or the body pattern is generated by subsequent cell-cell interaction [5]. The asymmetry of Xenopus egg endows the early blastomeres with different characters according to whether they are dorsal or ventral [6] [7]. However, it is also indicated that the blastomeres must be interacted with one another to generate the full range of cell type, by focusing on some special receptors [8] [9], other proteins [10] [11] [12], RHAs [13] and genes [14] [15]. The patterning of Drosophila embryo begins with the influence of the cells surrounding the egg [16] [17] and is investigated from the aspect of protein changes [18]. In contrast to the frog and fly eggs, the mammalian egg is essentially symmetrical, and all cells of the early mammalian embryo have the same developmental potential [19] [20]. Thus, the experiments of mammals first focus on 4- and 8-cell stage [21], clonal analysis [22], culture [23] and chimeras [24], then focus on the stem cells [25] [26] to find how environmental cues control the pace as well as the pathway of development, and gradually shift to gene targeting [27].

The recent genome sequencing reveals a notable feature that the cell of a multicellular eukaryote carries the enlarged repertoire of genes of proteins such as receptors, protein kinases and those for cell adhesion and most of differentiated cells still carry the same genome [28] [29] [30] [31], except for some special cells 
such as erythrocytes and immunocytes. Thus, the expression of specific genes must be regulated and controlled differently in differentiated cells. Moreover, many kinds of proteins including transcriptional regulators carry long stretches of special amino acid residue repeats in a eukaryote, in contrast to the prokaryote. Among such repeats, serine and threonine residues are known to be the sites for phosphorylation [32] and glycosylation [33] to change the activity of the protein. The transduction pathways of phosphorylation signal are partly followed experimentally from ligand-receptors to transcriptional regulators in some examples of differentiated cells [32] as well as to the cell-cycle control system in yeast [34] [35]. However, the transduction pathways are not simply linear relay chains but instead branch to activate many interacting components that operate in parallel, forming interconnected signal network. This complexity prevents the genetic and biochemical studies from outlining the essential future of cell differentiation, requiring a theoretical approach to the cell differentiation.

From the aspect of physics, it is also a curious problem how the cells having the same genome content differentiate into distinctive groups. The cell differentiation occurs in the assembly of a large number of cells after the cell proliferation, and this process is regarded as the stochastic process represented by the master equation in the present paper. The equation of the most probable path of cell assembly is mathematically derived from the master equation in which the transition of cells between proliferation and differentiation modes, the long-range interaction between the cells in different regions and the short-range interaction between the cells in the same region are considered. The equation of the most probable path obtained thus not only reproduces the cell differentiation but also explains most of experimental results concerning the cell differentiation, indicating the important role of the short-range interaction as well as the long-range interaction caused by ligand-receptor relationship in the cell differentiation.

\section{A Mathematical Model of Cell Differentiation}

A fertilized egg first proliferates using the material and energy source, with which the female parent has endowed. As the number of cells increases, the cell differentiation occurs. For simplicity, the total number of cells in an embryo is assumed to be constant during the process of differentiation in the present formulation. Then, the formulation of cell differentiation starts from the following master equation of the probability $P\left(N_{I^{+}}, N_{I-}, N_{I+}, N_{I I-}, N_{0} ; t\right)$, which we find $N_{I^{+}}$ cells of + type and $N_{I-}$ cells of - type in region $I, N_{I I+}$ cells of + type and $N_{I-}$ cells of - type in region $I I$, and $N_{0}$ undifferentiated cells at time $t$,

$$
\begin{aligned}
& \frac{\partial}{\partial t} P\left(N_{I+}, N_{I-}, N_{I I+}, N_{I I-}, N_{0} ; t\right) \\
& =-\left\{W\left(N_{I+}, N_{I-}, N_{0} \rightarrow N_{I+}+1, N_{I-}, N_{0}-1\right)+W\left(N_{I+}, N_{I-}, N_{0} \rightarrow N_{I+}, N_{I-}+1, N_{0}-1\right)\right. \\
& \quad+W\left(N_{I+}, N_{I-}, N_{0} \rightarrow N_{I+}-1, N_{I-}, N_{0}+1\right)+W\left(N_{I+} N_{I-}, N_{0} \rightarrow N_{I+}, N_{I-}-1, N_{0}+1\right) \\
& \quad+W\left(N_{I I+}, N_{I I-}, N_{0} \rightarrow N_{I I+}+1, N_{I I-}, N_{0}-1\right)+W\left(N_{I I+}, N_{I I-}, N_{0} \rightarrow N_{I I+}, N_{I I-}+1, N_{0}-1\right) \\
& \quad+W\left(N_{I I+}, N_{I I-}, N_{0} \rightarrow N_{I I+}-1, N_{I I-}, N_{0}+1\right)+W\left(N_{I I+}, N_{I I-}, N_{0} \rightarrow N_{I I+}, N_{I I-}-1, N_{0}+1\right)
\end{aligned}
$$




$$
\begin{aligned}
& +W\left(N_{I+}, N_{I-}, N_{0} \rightarrow N_{I+}+1, N_{I-}-1, N_{0}\right)+W\left(N_{I+}, N_{I-}, N_{0} \rightarrow N_{I+}-1, N_{I-}+1, N_{0}\right) \\
& \left.+W\left(N_{I I+}, N_{I I-}, N_{0} \rightarrow N_{I I+}+1, N_{I I-}-1, N_{0}\right)+W\left(N_{I I+}, N_{I I-}, N_{0} \rightarrow N_{I I+}-1, N_{I I-}+1, N_{0}\right)\right\} \\
& \times P\left(N_{I+}, N_{I-}, N_{I I+}, N_{I I-}, N_{0} ; t\right) \\
& +W\left(N_{I+}+1, N_{I-}, N_{0}-1 \rightarrow N_{I+}, N_{I-}, N_{0}\right) P\left(N_{I+}+1, N_{I-}, N_{I I+}, N_{I I-}, N_{0}-1 ; t\right) \\
& +W\left(N_{I+}, N_{I-}+1, N_{0}-1 \rightarrow N_{I+}, N_{I-}, N_{0}\right) P\left(N_{I+}, N_{I-}+1, N_{I I+}, N_{I I-}, N_{0}-1 ; t\right) \\
& +W\left(N_{I+}-1, N_{I-}, N_{0}+1 \rightarrow N_{I+}, N_{I-}, N_{0}\right) P\left(N_{I+}-1, N_{I-}, N_{I I+}, N_{I I-}, N_{0}+1 ; t\right) \\
& +W\left(N_{I+}, N_{I-}-1, N_{0}+1 \rightarrow N_{I+}, N_{I-}, N_{0}\right) P\left(N_{I+}, N_{I-}-1, N_{I I+}, N_{I I-}, N_{0}+1 ; t\right) \\
& +W\left(N_{I I+}+1, N_{I I-}, N_{0}-1 \rightarrow N_{I I+}, N_{I I-}, N_{0}\right) P\left(N_{I+}, N_{I-}, N_{I I+}+1, N_{I I-}, N_{0}-1 ; t\right) \\
& +W\left(N_{I I+}, N_{I I-}+1, N_{0}-1 \rightarrow N_{I I+}, N_{I I-}, N_{0}\right) P\left(N_{I+} N_{I-}, N_{I I+}, N_{I I-}+1, N_{0}-1 ; t\right) \\
& +W\left(N_{I I+}-1, N_{I I-}, N_{0}+1 \rightarrow N_{I I+}, N_{I I-}, N_{0}\right) P\left(N_{I+}, N_{I-}, N_{I I+}-1, N_{I I-}, N_{0}+1 ; t\right) \\
& +W\left(N_{I I+}, N_{I I-}-1, N_{0}+1 \rightarrow N_{I++}, N_{I I-}, N_{0}\right) P\left(N_{I+}, N_{I-}, N_{I I+}, N_{I I-}-1, N_{0}+1 ; t\right) \\
& +W\left(N_{I+}+1, N_{I-}-1, N_{0} \rightarrow N_{I+}, N_{I-}, N_{0}\right) P\left(N_{I+}+1, N_{I-}-1, N_{I I+}, N_{I I-}, N_{0} ; t\right) \\
& +W\left(N_{I+}-1, N_{I-}+1, N_{0} \rightarrow N_{I+} N_{I-}, N_{0}\right) P\left(N_{I+}-1, N_{I-}+1, N_{I I+}, N_{I I-}, N_{0} ; t\right) \\
& +W\left(N_{I I+}+1, N_{I I-}-1, N_{0} \rightarrow N_{I I+}, N_{I I-}, N_{0}\right) P\left(N_{I+}, N_{I-}, N_{I I+}+1, N_{I I-}-1, N_{0} ; t\right) \\
& +W\left(N_{I I+}-1, N_{I I-}+1, N_{0} \rightarrow N_{I I+}, N_{I I-}, N_{0}\right) P\left(N_{I+}, N_{I-}, N_{I I+}-1, N_{I I-}+1, N_{0}: t\right)
\end{aligned}
$$

Here, $W\left(N_{I+}, N_{I-}, N_{0} \rightarrow N_{I+}+1, N_{I-}, N_{0}-1\right)$ is the transition probability for an undifferentiated cell to change into +type in region $I$ and the other transition probabilities $W$ s are also used by denoting the changes in parentheses. These transition probabilities on the right-hand side of Equation (1) are explicitly expressed by the first four, ninth and tenth, thirteenth to sixteenth, and twenty-first and twenty-second transition probabilities in the following way.

$$
\begin{aligned}
& W\left(N_{I+}, N_{I-}, N_{0} \rightarrow N_{I+}+1, N_{I-}, N_{0}-1\right) \\
&= N_{0} \exp \left[\left\{\mu_{I}+\left(z_{I} J_{I} / 2 N\right)\left(N_{I+}-N_{I-}\right)-(f / 2 N)\left(N_{I++}-N_{I I-}\right)\right\} / k T\right] \\
& W\left(N_{I+}, N_{I-}, N_{0} \rightarrow N_{I+}, N_{I-}+1, N_{0}-1\right) \\
&= N_{0} \exp \left[\left\{\mu_{I}-\left(z_{I} J_{I} / 2 N\right)\left(N_{I+}-N_{I-}\right)+(f / 2 N)\left(N_{I++}-N_{I I-}\right)\right\} / k T\right] \\
& W\left(N_{I+}, N_{I-}, N_{0} \rightarrow N_{I+}-1, N_{I-}, N_{0}+1\right) \\
&= N_{I+} \exp \left[\left\{-\mu_{I}-\left(z_{I} J_{I} / 2 N\right)\left(N_{I+}-N_{I-}\right)+(f / 2 N)\left(N_{I++}-N_{I I-}\right)\right\} / k T\right] \\
& W\left(N_{I+}, N_{I-}, N_{0} \rightarrow N_{I+}, N_{I-}-1, N_{0}+1\right) \\
&= N_{I-} \exp \left[\left\{-\mu_{I}+\left(z_{I} J_{I} / 2 N\right)\left(N_{I+}-N_{I-}\right)-(f / 2 N)\left(N_{I I+}-N_{I I-}\right)\right\}\right] / k T \\
& W\left(N_{I+}, N_{I-}, N_{0} \rightarrow N_{I+}+1, N_{I-}-1, N_{0}\right) \\
&=N_{I-} \exp \left[\left\{\left(z_{I} J_{I} / 2 N\right)\left(N_{I+}-N_{I-}\right)-(f / N)\left(N_{I++}-N_{I I-}\right)\right\} / k T\right] \\
& W\left(N_{I+} N_{I-}, N_{0} \rightarrow N_{I+}-1, N_{I-}+1, N_{0}\right) \\
&= N_{I+} \exp \left[\left\{-\left(z_{I} J_{I} / N\right)\left(N_{I+}-N_{I-}\right)+(f / N)\left(N_{I++}-N_{I I-}\right)\right\} / k T\right] \\
& W\left(N_{I+}+1, N_{I-}, N_{0}-1 \rightarrow N_{I+} N_{I-}, N_{0}\right) \\
&=\left(N_{I+}\right) \exp \left[\left\{-\mu_{I}-\left(z_{I} J_{I} / 2 N\right)\left(N_{I+}+1-N_{I-}\right)+(f / 2 N)\left(N_{I I+}-N_{I I-}\right)\right\} / k T\right]
\end{aligned}
$$




$$
\begin{aligned}
W & \left(N_{I+}, N_{I-}+1, N_{0}-1 \rightarrow N_{I+}, N_{I-}, N_{0}\right) \\
= & \left(N_{I-}+1\right) \exp \left[\left\{-\mu_{I}+\left(z_{I} J_{I} / 2 N\right)\left(N_{I+}-N_{I-}-1\right)-(f / 2 N)\left(N_{I I+}-N_{I I-}\right)\right\} / k T\right] \\
& W\left(N_{I+}-1, N_{I-}, N_{0}+1 \rightarrow N_{I+}, N_{I-}, N_{0}\right) \\
= & \left(N_{0}+1\right) \exp \left[\left\{\mu_{I}+\left(z_{I} J_{I} / 2 N\right)\left(N_{I+}-1-N_{I-}\right)-(f / 2 N)\left(N_{I I+}-N_{I I-}\right)\right\} / k T\right] \\
W & \left(N_{I+}, N_{I-}-1, N_{0}+1 \rightarrow N_{I+}, N_{I-}, N_{0}\right) \\
= & \left(N_{0}+1\right) \exp \left[\left\{\mu_{I}-\left(z_{I} J_{I} / 2 N\right)\left(N_{I+}-N_{I-}+1\right)+(f / 2 N)\left(N_{I I+}-N_{I I-}\right)\right\} / k T\right] \\
W & \left(N_{I+}+1, N_{I-}-1, N_{0} \rightarrow N_{I+}, N_{I-}, N_{0}\right) \\
= & \left(N_{I+}+1\right) \exp \left[\left\{-\left(z_{I} J_{I} / N\right)\left(N_{I+}-N_{I-}+2\right)+(f / N)\left(N_{I I+}-N_{I I-}\right)\right\} / k T\right] \\
& W\left(N_{I+}-1, N_{I-}+1, N_{0} \rightarrow N_{I+}, N_{I-}, N_{0}\right) \\
& =\left(N_{I-}+1\right) \exp \left[\left\{\left(z_{I} J_{I} / N\right)\left(N_{I+}-N_{I-}-2\right)-(f / N)\left(N_{I+}-N_{I I-}\right)\right\} / k T\right]
\end{aligned}
$$

By exchanging the subscripts from $I$ to $I I$ and from $I I$ to $I$, the fifth to eighth transition probabilities $W\left(N_{I I+}, N_{I I-}, N_{0} \rightarrow N_{I I+}+1, N_{I I-}, N_{0}-1\right) \sim$ $W\left(N_{I I+}, N_{I I-}, N_{0} \rightarrow N_{I I+}, N_{I I-}-1, N_{0}+1\right)$ are expressed from Equations -(2-4), the eleventh and twelfth transition probabilities

$W\left(N_{I I+}, N_{I I-}, N_{0} \rightarrow N_{I I+}+1, N_{I I-}-1, N_{0}\right)$ \& $W\left(N_{I I+}, N_{I I-}, N_{0} \rightarrow N_{I I+}-1, N_{I I-}+1, N_{0}\right)$ are expressed from Equations (2-5) and (2-6), respectively, the seventeenth to twentieth transition probabilities $W\left(N_{I I+}+1, N_{I I-}, N_{0}-1 \rightarrow N_{I I+}, N_{I I-}, N_{0}\right) \sim$ $W\left(N_{I I+}, N_{I I-}-1, N_{0}+1 \rightarrow N_{I I+}, N_{I I-}, N_{0}\right)$ are expressed from Equations (2-7) -(2-10), and the twenty-third and the last transition probabilities

$W\left(N_{I I+}+1, N_{I I-}-1, N_{0} \rightarrow N_{I I+}, N_{I I-}, N_{0}\right)$ \& $W\left(N_{I I+}-1, N_{I I-}+1, N_{0} \rightarrow N_{I I+}, N_{I I-}, N_{0}\right)$ are expressed from Equations (2-11) and (2-12), respectively.

In the above expression, the transition probability from an undifferentiated cell to the differentiated state, + type or - type, in the region $I$ and that to differentiated state in the region $I I$ are denoted by $\exp \left(\mu_{I} / k T\right)$ and $\exp \left(\mu_{I I} / k T\right)$, respectively, using Boltzmann's constant $k$ and temperature $T$. The reverse transition probabilities from these differentiated cells to the undifferentiated cell are denoted by $\exp \left(-\mu_{I} / k T\right)$ and $\exp \left(-\mu_{I I} / k T\right)$, respectively. The terms with the coefficient $Z_{I} J_{I}$ and those with the coefficient $z_{I I} J_{I I}$ in the exponents indicate the short-range interaction between the cells in the region $I$ and that in the region $I$, respectively. The terms with the coefficient $f$ in the exponents indicate the long-range interaction between the cells in region $I$ and those in region $I I$. Using the Ising model [36] that the cell of + type takes the value of +1 and the cell of type takes the value of -1 , the cells in the same region tend to take the same type by the short-range interaction while the cells in region $I$ tend to take the type different from the type in region $I I$ by the long-range interaction.

These transition probabilities (2-1)-(2-12) and those with replaced subscripts $I$ by $I I$ and $I I$ by $I$ assure the following stationary distribution $P_{s t}\left(N_{I+}, N_{I-}, N_{I I+}, N_{I I}\right.$, $N_{0}$ ) when both $\mu_{I}$ and $\mu_{I I}$ are equal to $\mu$. 


$$
\begin{aligned}
& P_{s t}\left(N_{I+}, N_{I-}, N_{I I+}, N_{I I-}, N_{0}\right) \\
& =\frac{N !}{N_{I+} ! N_{I-} ! N_{I+} ! N_{0} !} \exp \left[\left\{\frac{z_{I} J_{I}}{2 N}\left(N_{I+}-N_{I-}\right)^{2}+\frac{z_{I I} J_{I I}}{2 N}\left(N_{I I+}-N_{I I-}\right)^{2}\right.\right. \\
& \left.\left.-\frac{f}{N}\left(N_{I+}-N_{I-}\right)\left(N_{I I+}-N_{I I-}\right)+\mu\left(N_{I+}+N_{I-}+N_{I I+}+N_{I I-}-N_{0}\right)\right\} / k T\right]
\end{aligned}
$$

The probability that the transitions (2-1)-(2-12) and those with the replaced subscripts take place in an infinitely short time interval is assumed to be proportional to the total number $N$ of cells. Then, the probability density function $p\left(X_{I}\right.$ $\left.X_{I}, X_{I J}, X_{I J}, t\right)$ is defined by

$$
p\left(X_{I}, x_{I}, X_{I I}, x_{I I} ; t\right)=\varepsilon P\left(N_{I+}, N_{I-}, N_{I I+}, N_{I I-}, N_{0} ; t\right)
$$

Here, $\varepsilon$ is the inverse of system size defined by $1 / N$, and the new variables $X_{I}, X_{I I}$, $x_{I}$ and $x_{I I}$ are defined by

$$
X_{I}=\frac{N_{I+}+N_{I-}}{N}, X_{I I}=\frac{N_{I I+}+N_{I I+}}{N}, x_{I}=\frac{N_{I+}-N_{I-}}{N}, x_{I I}=\frac{N_{I I+}-N_{I I-}}{N}
$$

With this assumption and the new variables, the master Equation (1) is rewritten into the following equation of probability density function $p\left(X_{I}, X_{I}, X_{I I}, X_{I I}, t\right)$;

$$
\begin{aligned}
& \frac{\partial}{\partial t} p\left(X_{I}, x_{I}, X_{I I}, x_{I I} ; t\right) \\
&=\sum_{n=1}^{\infty} \frac{(-1)^{n}}{n !} \varepsilon^{n-1}\left[\left(\frac{\partial}{\partial X_{I}}+\frac{\partial}{\partial x_{I}}\right)^{n} \frac{(-1)^{n}}{2}\left(X_{I}+x_{I}\right) \exp \left(-\alpha_{I}-\beta_{I} x_{I} / 2+\gamma x_{I I} / 2\right)\right. \\
&+\left(\frac{\partial}{\partial X_{I}}-\frac{\partial}{\partial x_{I}}\right)^{n} \frac{(-1)^{n}}{2}\left(X_{I}-x_{I}\right) \exp \left(-\alpha_{I}+\beta_{I} x_{I} / 2-\gamma x_{I I} / 2\right) \\
&+\left(\frac{\partial}{\partial X_{I}}+\frac{\partial}{\partial x_{I}}\right)^{n}\left(1-X_{I}-X_{I I}\right) \exp \left(\alpha_{I}+\beta_{I} x_{I} / 2-\gamma x_{I I} / 2\right) \\
&+\left(\frac{\partial}{\partial X_{I}}-\frac{\partial}{\partial x_{I}}\right)^{n}\left(1-X_{I}-X_{I I}\right) \exp \left(\alpha_{I}-\beta_{I} x_{I} / 2+\gamma x_{I I} / 2\right) \\
&+\left(\frac{\partial}{\partial X_{I I}}+\frac{\partial}{\partial x_{I I}}\right)^{n} \frac{(-1)^{n}}{2}\left(X_{I I}+x_{I I}\right) \exp \left(-\alpha_{I I}-\beta_{I I} x_{I I} / 2+\gamma x_{I} / 2\right) \\
&+\left(\frac{\partial}{\partial X_{I I}}-\frac{\partial}{\partial x_{I I}}\right)^{n} \frac{(-1)^{n}}{2}\left(X_{I I}-x_{I I}\right) \exp \left(-\alpha_{I I}+\beta_{I I} x_{I I} / 2-\gamma x_{I} / 2\right) \\
&+\left(\frac{\partial}{\partial X_{I I}}+\frac{\partial}{\partial x_{I I}}\right)^{n}\left(1-X_{I}-X_{I I}\right) \exp \left(\alpha_{I I}+\beta_{I I} x_{I I} / 2-\gamma x_{I} / 2\right) \\
&+\left(\frac{\partial}{\partial X_{I I}}-\frac{\partial}{\partial x_{I I}}\right)^{n}\left(1-X_{I}-X_{I I}\right) \exp \left(\alpha_{I I}-\beta_{I I} x_{I I} / 2+\gamma x_{I} / 2\right) \\
&+\left(-2 \frac{\partial}{\partial x_{I}}\right)^{n}\left(X_{I}+x_{I}\right) \exp \left(-\beta_{I} x_{I}+\gamma x_{I I}\right) \\
&+\left(2 \frac{\partial}{\partial x_{I}}\right)^{n}\left(X_{I}-x_{I}\right) \exp \left(\beta_{I} x_{I}-\gamma x_{I I}\right)
\end{aligned}
$$




$$
\begin{aligned}
& +\left(-2 \frac{\partial}{\partial x_{I I}}\right)^{n}\left(X_{I I}+x_{I I}\right) \exp \left(-\beta_{I I} x_{I I}+\gamma x_{I}\right) \\
& \left.+\left(2 \frac{\partial}{\partial x_{I I}}\right)^{n}\left(X_{I I}-x_{I I}\right) \exp \left(\beta_{I I} x_{I I}-\gamma x_{I}\right)\right] p\left(X_{I}, x_{I}, X_{I I}, x_{I I} ; t\right)
\end{aligned}
$$

Here, the quantities $\mu_{I} / k T, \mu_{I I} / k T, z_{I} J_{I} / k T, z_{I I} J_{I I} / k T$ and $f k T$ are simply denoted as $\alpha_{I}, \alpha_{I I}, \beta_{I}, \beta_{I I}$ and $\gamma$, respectively.

As shown already by the expansion method for proving the central limit theorem [37] and more generally by the cumulant expansion method for the characteristic function of the probability density function [38], the probability density function can be expressed as the following Gaussian form

$$
\begin{aligned}
& p\left(X_{I}, x_{I}, X_{I I}, x_{I I} ; t\right) \\
& \propto \exp \left[-\frac{1}{2 \varepsilon}\left\{\sigma_{X I X I}^{-1}\left(X_{I}-Y_{I}\right)^{2}+\sigma_{X I X I I}^{-1}\left(X_{I}-Y_{I}\right)\left(X_{I I}-Y_{I I}\right)\right.\right. \\
& +\sigma_{X I X I I}^{-1}\left(X_{I I}-Y_{I I}\right)^{2}+\sigma_{x I X I}^{-1}\left(x_{I}-y_{I}\right)\left(X_{I}-Y_{I}\right)+\sigma_{x I X I I}^{-1}\left(x_{I}-y_{I}\right)\left(X_{I I}-Y_{I I}\right) \\
& +\sigma_{X I X I I}^{-1}\left(x_{I I}-y_{I I}\right)\left(X_{I}-Y_{I}\right)+\sigma_{X I X I I}^{-1}\left(x_{I I}-y_{I I}\right)\left(X_{I I}-Y_{I I}\right) \\
& \left.\left.+\sigma_{X I X I}^{-1}\left(x_{I}-y_{I}\right)^{2}+\sigma_{X X X I I}^{-1}\left(x_{I}-y_{I}\right)\left(x_{I I}-y_{I I}\right)+\sigma_{X I X X I I}^{-1}\left(x_{I I}-y_{I I}\right)^{2}\right\}\right]
\end{aligned}
$$

using the elements of inverse matrix $\sigma^{-1}$ of standard deviation matrix $\sigma$, when the value of $\varepsilon$ is very small or the number of $N$ is very large and the right-hand side of Equation (6) can be approximately terminated at the second moments, i.e.,

$$
\begin{aligned}
& \frac{\partial}{\partial t} p\left(X_{I}, x_{I}, X_{I I}, x_{I I} ; t\right) \\
= & \left\{-\left(\frac{\partial}{\partial X_{I}} m_{1 X I}+\frac{\partial}{\partial X_{I I}} m_{1 X I I}+\frac{\partial}{\partial x_{I}} m_{1 \times I}+\frac{\partial}{\partial x_{I I}} m_{1 X I I}\right)\right. \\
& +\frac{1}{2} \varepsilon\left(\frac{\partial^{2}}{\partial X_{I}^{2}} m_{2 X I X I}+2 \frac{\partial^{2}}{\partial X_{I} \partial x_{I}} m_{2 X I X 1}+\frac{\partial}{\partial x_{I}^{2}} m_{2 \times I I I}+\frac{\partial^{2}}{\partial X_{I I}^{2}} m_{2 X I I X I I}\right. \\
& \left.\left.+2 \frac{\partial^{2}}{\partial x_{I I} \partial X_{I I}} m_{2 X I I X I I}+\frac{\partial^{2}}{\partial x_{I I}^{2}} m_{2 \times I I X I I}\right)+\cdots\right\} p\left(X_{I}, x_{I}, X_{I I}, x_{I I} ; t\right)
\end{aligned}
$$

The time changes in the most probable values $Y_{I}, Y_{I I}, y_{I}$ and $y_{I I}$ in the Gaussian distribution (7) are determined by the first moments of the transition probabilities $m_{1 X I}, m_{I X I}, m_{I X I}$ and $m_{1 X I I}$ in Equation (8), respectively, as follows.

$$
\begin{aligned}
& \frac{\mathrm{d}}{\mathrm{d} t} Y_{I}=m_{1 X I}\left(Y_{I}, Y_{I I} ; y_{I}, y_{I I} ; \alpha_{I}\right) \\
& =-Y_{I} \mathrm{e}^{-\alpha I} \cosh \left(\beta_{I} y_{I} / 2-\gamma y_{I I} / 2\right)+y_{I} \mathrm{e}^{-\alpha I} \sinh \left(\beta_{I} y_{I} / 2-\gamma y_{I I} / 2\right) \\
& \quad+2\left(1-Y_{I}-Y_{I I}\right) \mathrm{e}^{\alpha I} \cosh \left(\beta_{I} y_{I} / 2-\gamma y_{I I} / 2\right) \\
& \frac{\mathrm{d}}{\mathrm{d} t} Y_{I I}=m_{1 X I I}\left(Y_{I I}, Y_{I} ; y_{I I}, y_{I}, \alpha_{I I}\right) \\
& =-Y_{I I} \mathrm{e}^{-\alpha I I} \cosh \left(\beta_{I I} y_{I I} / 2-\gamma y_{I} / 2\right)+y_{I I} \mathrm{e}^{-\alpha I I} \sinh \left(\beta_{I I} y_{I I} / 2-\gamma y_{I} / 2\right) \\
& +2\left(1-Y_{I}-Y_{I I}\right) \mathrm{e}^{\alpha I I} \cosh \left(\beta_{I I} y_{I I} / 2-\gamma y_{I} / 2\right)
\end{aligned}
$$




$$
\begin{aligned}
\frac{\mathrm{d}}{\mathrm{d} t} y_{I}= & m_{1 x I}\left(y_{I}, y_{I I} ; Y_{I}, Y_{I I} ; \alpha_{I}\right) \\
= & Y_{I} \mathrm{e}^{-\alpha I} \sinh \left(\beta_{I} y_{I} / 2-\gamma y_{I I} / 2\right)-y_{I} \mathrm{e}^{-\alpha I} \cosh \left(\beta_{I} y_{I} / 2-\gamma y_{I I} / 2\right) \quad(9-3) \\
& +2\left(1-Y_{I}-Y_{I I}\right) \mathrm{e}^{\alpha I} \sinh \left(\beta_{I} y_{I} / 2-\gamma y_{I I} / 2\right) \\
& +4 Y_{I} \sinh \left(\beta_{I} y_{I}-\gamma y_{I I}\right)-4 y_{I} \cosh \left(\beta_{I} y_{I}-\gamma y_{I I}\right) \\
\frac{\mathrm{d}}{\mathrm{d} t} y_{I I}= & m_{1 x I I}\left(y_{I I}, y_{I} ; Y_{I I}, Y_{I} ; \alpha_{I I}\right) \\
= & Y_{I I} \mathrm{e}^{-\alpha I I} \sinh \left(\beta_{I I} y_{I I} / 2-\gamma y_{I} / 2\right)-y_{I I} \mathrm{e}^{-\alpha I I} \cosh \left(\beta_{I I} y_{I I} / 2-\gamma y_{I} / 2\right)(9-4) \\
& +2\left(1-Y_{I}-Y_{I I}\right) \mathrm{e}^{\alpha I I} \sinh \left(\beta_{I I} y_{I I} / 2-\gamma y_{I} / 2\right) \\
& +4 Y_{I I} \sinh \left(\beta_{I I} y_{I I}-\gamma y_{I}\right)-4 y_{I I} \cosh \left(\beta_{I I} y_{I I}-\gamma y_{I}\right)
\end{aligned}
$$

Equations (9-1)-(9-4) well reproduce the cell differentiation, although this set of equations is derived under the constant number of total cells and is not sufficient for representing the developmental process where cell proliferation and differentiation progress in parallel. When $\alpha_{I}$ and $\alpha_{I I}$ take sufficiently large positive values, the smaller second term on the right-hand side of Equation (9-1) is neglected, and $\mathrm{d} Y_{I} / \mathrm{d} t=0$ approximately holds for the following relation between $Y_{I s t}$ and $Y_{I I s t}$

$$
Y_{\text {Ist }} \approx 2\left(1-Y_{\text {Ist }}-Y_{\text {IIst }}\right) \mathrm{e}^{2 \alpha I}
$$

By the similar way on Equation (9-2), $\mathrm{d} Y_{I I} / \mathrm{d} t=0$ approximately holds for the following relation between $Y_{I I s t}$ and $Y_{\text {Ist }}$

$$
Y_{\text {IIst }} \approx 2\left(1-Y_{\text {Ist }}-Y_{\text {IIst }}\right) \mathrm{e}^{2 \alpha I I}
$$

From these relations, $Y_{I s t}$ and $Y_{\text {IIst }}$ in the stationary state are expressed as

$$
\begin{aligned}
Y_{I s t} & =\frac{2 \mathrm{e}^{2 \alpha I}}{1+2 \mathrm{e}^{2 \alpha I}+2 \mathrm{e}^{2 \alpha I I}} \\
Y_{I I S t} & =\frac{2 \mathrm{e}^{2 \alpha I I}}{1+2 \mathrm{e}^{2 \alpha I}+2 \mathrm{e}^{2 \alpha I I}}
\end{aligned}
$$

Here, the quantity $1-Y_{\text {Ist }}-Y_{\text {IIst }}$ corresponds to the ratio of undifferentiated cells or stem cells. In this stationary state, the first, second and third terms on the right-hand side of Equation (9-3) are neglected because $2\left(1-Y_{\text {Ist }}-Y_{\text {IIst }}\right) \mathrm{e}^{\alpha_{I}}$ in the third term is equal to $Y_{I s t} \mathrm{e}^{-\alpha_{I}}$, and $\mathrm{d} y_{I} / \mathrm{d} t=0$ holds when the fourth and fifth terms are equal to zero, i.e.,

$$
Y_{\text {Ist }} \sinh \left(\beta_{I} y_{I}-\gamma y_{I I}\right)-y_{I} \cosh \left(\beta_{I} y_{I}-\gamma y_{I I}\right) \approx 0
$$

The solution of $y_{I}$ satisfying Equation (12-1) is obtained by the following graphical procedure. When a new quantity $u_{I}$ is introduced by

$$
u_{I} \equiv \beta_{I} y_{I}-\gamma y_{I I}
$$

Equation (12-1) is rewritten into

$$
y_{I}=Y_{I s t} \tanh u_{I}
$$

As shown in Figure 1(a), the value of $y_{I s t}$ is obtained as the ordinate of the crossing point of the curve (12-3) with the straight line (12-2) concerning $y_{I}$ values 
(a)

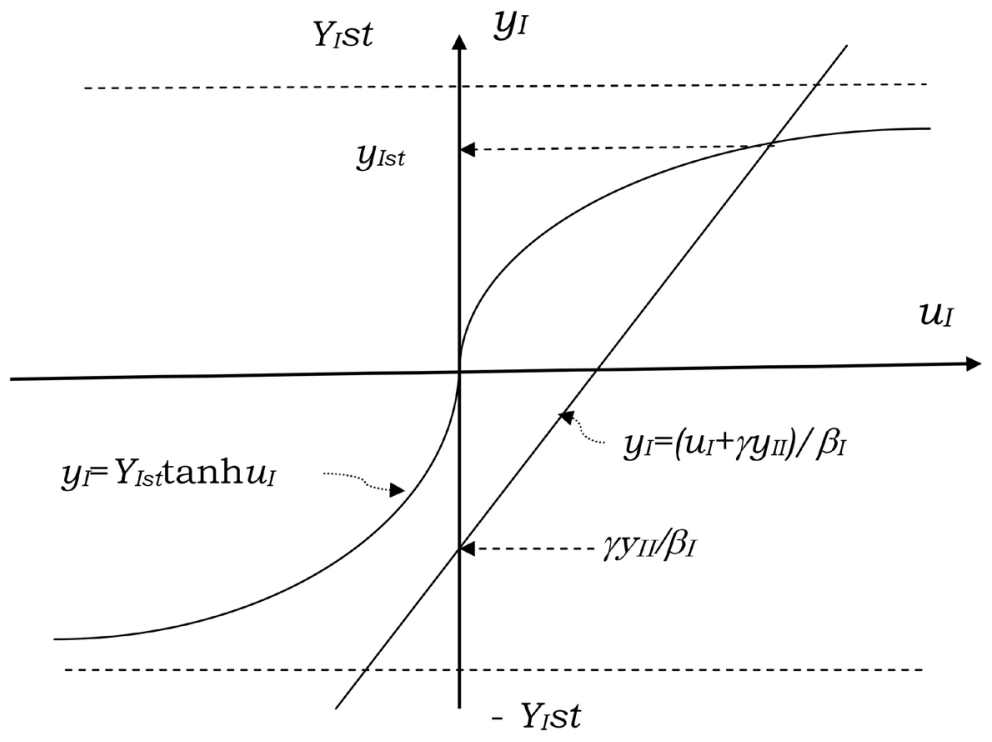

(b)

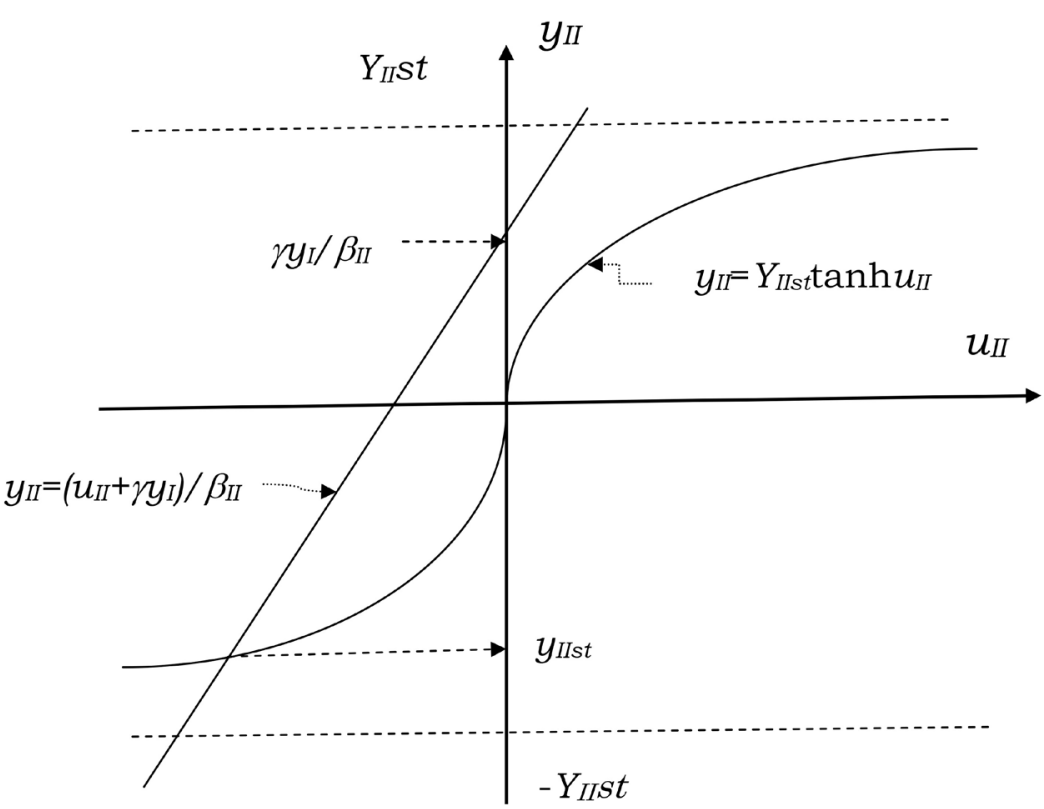

Figure 1. The graphical procedure to obtain the ratios $y_{I s t}$ and yIst $_{\text {of }}$ of differentiated cells. (a) The values of $y_{I}$ are plotted against $u_{I}$ values according to Equations (12-2) and (12-3). The value of $y_{\text {Ist }}$ satisfying Equation (12-1) is obtained as the ordinate of the crossing point of the curve (12-3) with the straight line (12-2). If $y_{I I}$ is chosen to be a negative value, this value of $y_{I s t}$ becomes positive as shown in the figure. Moreover, this result also indicates that the value of $y_{I s t}$ becomes larger, nearer to $Y_{I}$, as the short-range and long-range interaction parameters, $\beta_{I}$ and $\gamma$, take larger values, respectively. (b) The values of $y_{I I}$ are plotted against $u_{I I}$ values according to Equations (13-2) and (13-3). The value of $y_{I I s t}$ satisfying Equation (13-1) is obtained as the ordinate of the crossing point of the curve (13-3) and the straight line (13-2). When $y_{I}$ is chosen to be a positive value, this value of $y_{I I s t}$ becomes negative, consistent with the result of $(\alpha)$. The value of such $y_{I S t}$ becomes nearer to that of $-Y_{I I}$ as the short-range and long-range interaction parameters, $\beta_{I I}$ and $\gamma$, take the larger values, respectively. Thus, the differentiation into + type of cells in region $I$ and - types of cells in region $I I$ occurs under both the long-range and short-range interactions. 
plotted against $u_{I}$ values. This value of $y_{I s t}$ becomes positive if $y_{I I}$ is chosen to be a negative value. In Equation (9-4), $\mathrm{d} y_{I I} / \mathrm{d} t=0$ approximately holds when the fourth and fifth terms on the right-hand side are equal to zero, i.e.,

$$
Y_{I I s t} \sinh \left(\beta_{I I} y_{I I}-\gamma y_{I}\right)-y_{I I} \cosh \left(\beta_{I I} y_{I I}-\gamma y_{I}\right) \approx 0
$$

The solution of $y_{I I}$ satisfying Equation (13-1) is also obtained by the following graphical method. By setting

$$
u_{I I} \equiv \beta_{I I} y_{I I}-\gamma y_{I}
$$

Equation (13-1) is rewritten into

$$
y_{I I}=Y_{I I s t} \tanh u_{I I}
$$

As seen in Figure 1(b), the ordinate $y_{I I s t}$ as the crossing point of the curve $y_{I I}$ vs $u_{I I}$ (13-3) with the straight line (13-2) becomes a negative value when $y_{I}$ takes a positive value. This is consistent with the result of Equations (12-2) and (12-3). Thus, it is shown theoretically that at least two types of interaction between cells, the short-range interaction $\left(\beta_{I}, \beta_{I I}>0\right)$ and the long-range interaction $(\gamma>0)$, reproduce the cell differentiation.

The elements of the standard deviation in the Gaussian distribution (7) are also derived from the present scheme. For example, the element $\sigma_{x I x I}$ of standard deviation changes with time by the following equation.

$$
\begin{aligned}
\frac{\mathrm{d}}{\mathrm{d} t} \sigma_{x I X I}= & 2 \frac{\partial m_{1 \times I}}{\partial y_{I}} \sigma_{x I X I}+\frac{\partial m_{1 x I}}{\partial y_{I I}}\left(\sigma_{x I X I I}+\sigma_{x I I X I}\right)+\frac{\partial m_{1 x I}}{\partial Y_{I}}\left(\sigma_{x I X I}+\sigma_{X I X I}\right) \\
& +\frac{\partial m_{1 x I}}{\partial Y_{I I}}\left(\sigma_{x I X I I}+\sigma_{X I X X I}\right)+m_{2 x I X I}
\end{aligned}
$$

This element becomes larger in the middle stage of cell differentiation but it slows down to a smaller value when the differentiation is accomplished. The other elements of standard deviation also show the similar behaviour. However, the more detailed discussion on the standard deviation will be reserved until the experimental data of standard deviation upon cell differentiation are accumulated.

Although the present model is restricted to the differentiation into two types of cells for simplicity, it can be extended to the higher hierarchy of cell differentiation, e. g. the + type of cells further differentiate into ++ and +- types of cells and the - type of cells further differentiate into -+ type and -- type of cells.

\section{Discussion}

In the present paper, it is shown theoretically that both long-range interaction and short-range interaction are necessary for cell differentiation. The long-range interaction is probably caused by the special ligand-receptor relationship. For example, the + type of cell secretes the ligand a, which is accepted by the receptor $A$ on the surface of the - type of cell, while the - type of cell secretes the ligand $b$ to be accepted by the receptor $B$ on the surface of the + type of cell. The receptor $A$ having accepted the ligand $a$ induces the expression of the genes to 
characterize the - type of cell through the intracellular signal transduction to the transcriptional regulators. On the other hand, the receptor $B$ having accepted the ligand $b$ induces the intracellular signal transduction to the transcriptional regulators which express the genes characteristic to the + type of cell. Although the molecular events underlying the short-range interaction is not clarified yet by biochemical studies, the contact between the same type of cells, probably through the cytoskeletons in the respective cells, may stabilize the intracellular signal transduction induced by the ligand-receptor relationship. Some types of differentiated cells such as erythrocyte and immunocyte are not in contact, but their generation is carried out in the state of contact. With the above molecular events in mind, the present model explains the following experimental results (1) - (4) on the cell differentiation.

1) Organizer

The organizer found in embryo may be the initial process of cell differentiation to form the long-range interaction. The cells in region $I$, for example, first begin to express the gene(s) of ligand as well as of receptor $B$. Although the receptor $A$ is spontaneously expressed in the cells of region $I I$ at this stage and its lifetime is relatively short, the receptor $A$ having accepted ligand a becomes stable enough to form the intracellular signal transduction to express the genes characteristic to the - type cell. These expressed genes include the gene of ligand $b$ which is to be accepted by the receptor $B$ on the surface of cells in region $I$ to exhibit the character of + type. In this case, the cells in region $I$ correspond to the (primary) organizer and the cells in region $I I$ correspond to the cells forming the induced organ. In the case when the cells constituting the induced organ further express the genes of ligand $c$ as well as of ligand $b$, the induced organ becomes the secondary organizer. Although the cells in region $I$ and those in regions $I I$ are assumed to be symmetric in the present model for generality, the preferential expression of specific gene(s) of ligand to rouse the long-range interaction may occur depending on the position of cells. In the embryo of animal, the ooplasmic segregation and/or moulding of proliferated cells may cause the difference in initial expression of genes between the cells in distinctive regions. In the germination of seed, the environmental difference of ground and light and/or the positional relation with albumen may cause the difference in gene expression depending on cell positions.

2) Memory

At the early stage of embryo, the removal of some cells hardly influences the growth of remaining cell assembly to the adult form. If the removed cells are transplanted to a new position in the embryo, they finally become the tissue depending on the new position. If the removal and transplantation are carried out at the later stage of embryo, on the contrary, the transplanted cells become the same tissue as the one generated in the original position. This phenomenon interpreted in terms of "field" and "determination" [3] can be explained by the present formulation; differentiated cells are memorized by the signal transduction through the long-range and short-range interactions. 
3) Tissue Culture

When the cells taken out from an animal tissue are separated from each other and cultured in the medium sufficient for nutrients, they gradually weaken the property characteristic to the original tissue and begin proliferation. This suggests a role of short-range interaction in stabilizing the cell differentiation. As the density of cultured cells is increased, however, the cultured cells considerably restore the property in the original tissue. This strongly suggests that the intracellular signal transduction formed in the original tissue hardly disappears even through the proliferation of cells.

4) ES cells and iPS cells

In the medical studies, it has been a long dream to obtain undifferentiated cells and to replace a diseased tissue by the new one induced from the undifferentiated cells. The first candidate for such undifferentiated cells is the embryonic stem (ES) cells. However, it is contrary to humanity to extract ES cells from human embryo and moreover their transplantation is confronted with the rejection of the recipient. Recently, a technological method to convert differentiated cells into undifferentiated ones is devised; the four kinds of genes, Oct3/4, Sox 2, $K I f 4$ and $c-M y c$, which are specifically expressed in the $E S$ cell, are injected into the fibroblast cells taken from the mouse, using the retrovirus vector, and such cells are cultured in the medium. After a few weeks, this method yields induced pluripotent stem (iPS) cells, which can be converted to various kinds of tissues or organs [39]. Subsequently, this method also succeeds in human cells [40]. This fact strongly suggests that the products expressed by the injected genes play the role not only in erasing the signals characteristic to the tissue cells but also in raising up the transition probability from differentiated mode to undifferentiated mode. This process is also contained in the result of the present model.

For this illustration, we consider the case when the cells are taken out from the region $I$ and the four kinds of genes are injected into these cells. For such cells, Equations (9-1) and (9-3) are evaluated in the following way; $\alpha_{I}$ takes a negative value and both $\beta_{I}$ and $\gamma$ become zero. Then, $\sinh \left(\beta_{I} y_{I}-\gamma y_{I I}\right)=0$, $\cosh \left(\beta_{I} y_{I}-\gamma y_{I I}\right)=1$ and the term containing $\mathrm{e}^{\alpha I}$ is neglected. Thus, Equations (9-1) and (9-3) are reduced to

$$
\frac{\mathrm{d}}{\mathrm{d} t} Y_{I}=-Y_{I} \mathrm{e}^{-\alpha I}
$$

and

$$
\frac{\mathrm{d}}{\mathrm{d} t} y_{I}=-y_{I} \mathrm{e}^{-\alpha I}-4 y_{I}
$$

respectively. These equations show that both $Y_{I}$ and $y_{I}$ become zero with time, indicating that the cells taken from the region $I$ become undifferentiated. This is also the case for the cells in region $I I$.

\section{Conclusion}

In this way, the present mathematical model not only reproduces the cell diffe- 
rentiation but also explains the experimental results concerning the cell differentiation, especially in animals. In particular, the present study proposes that future biochemical and genetic studies are directed to summarize the intercellular and intracellular signal transduction causing the cell differentiation into the long-rang interaction between distinctive types of cells and the short-range interaction between the same type of cells.

\section{Acknowledgements}

The author is particularly grateful to Professor Ryogo Kubo for his instructive discussions on a generalization of the Gaussian properties to non-equilibrium and non-stationary processes.

\section{Conflicts of Interest}

The author declares no conflicts of interest regarding the publication of this paper.

\section{References}

[1] Spemann, H. and Mangold, H. (1924) Über Induktion von Embryonalagen Durch Implantation artfrem der Organisatoren. Archiv für mikroskopiche Anatomie und Entwicklungsmechanik, 100, 599-638. https://doi.org/10.1007/BF02108133

[2] Spemann, H. (1938) Embryonic Development and Induction. Yale University Press, New Haven, CT.

[3] Waddington, C.H. (1957) Principles of Embryology. George Allen \& Unwin Ltd., London.

[4] Thom, R. (1983) Mathematical Models of Morphogenesis. Translated by Brookes, W. E. Ellis Horwood Limited, A Division of John Wiley \& Sons, New York, Brisbane, Chichester, Ontario.

[5] Gurdon, J.B. (1992) The Generation of Diversity and Pattern in Animal Development. Cell, 68, 185-199. https://doi.org/10.1016/0092-8674(92)90465-O

[6] Gerhart, J., Danilchik, M., Dooniach, T., Roberts, S., Rowning, B. and Stewart, R. (1989) Cortical Rotation of the Xenopus Egg: Consequences for the Anteroposterior Pattern of Embryonic Dorsal Development. Development, 107, 37-51.

[7] Horvitz, H.R. and Herskowitz, I. (1992) Mechanisms of Asymmetric Cell Division: Two Bs or Not Two Bs, That Is the Question. Cell, 68, 237-255. https://doi.org/10.1016/0092-8674(92)90468-R

[8] Amaya, E., Musci, T.J. and Kirschner, M.W. (1991) Expression of a Dominant Negative Mutant of the FGF Receptor Disrupts Mesoderm Formation in Xenopus Embryos. Cell, 66, 257-270. https://doi.org/10.1016/0092-8674(91)90616-7

[9] Hemmati-Brivanlou, A. and Melton, D.A. (1992) A Truncated Activin Receptor Inhibits Mesoderm Induction and Formation of Axial Structures in Xenopus Embryos. Nature, 359, 609-614. https://doi.org/10.1038/359609a0

[10] Dale, L., Howes, G., Price, B.M.J. and Smith, J.C. (1992) Bone Morphogenetic Protein 4: A Ventralizing Factor in Xenopus Development. Development, 115, 573-585.

[11] Smith, W.C., Knecht, A.K., Wu, M. and Harland, R.M. (1993) Secreted noggin Protein Mimics the Spemann Organizer in Dorsalizing Xenopus Mesoderm. Nature, 361, 547-549. https://doi.org/10.1038/361547a0 
[12] Thomsen, G.H. and Melton, D.A. (1993) Processed Vg1 Protein Is an Axial Mesoderm Inducer in Xenopus. Cell, 74, 433-441. https://doi.org/10.1016/0092-8674(93)80045-G

[13] Smith, W.C. and Harland, R.M. (1991) Injected Xwnt-8RNA Acts Early in Xenopus Embryos to Promote Formation of Vegetal Dorsalizing Center. Cell, 67, 753-765. https://doi.org/10.1016/0092-8674(91)90070-F

[14] Cho, K.W.Y., Blumberg, B., Steinbeisser, H. and De Robertis, E.M. (1991) Molecular Nature of Spemann's Organizer: The Role of the Xenopus Homeobox Gene goosecoid. Cell, 67, 1111-1120. https://doi.org/10.1016/0092-8674(91)90288-A

[15] Bae, C.J., Hong, C.S. and Saint-Jeanet J.P. (2018) Anosmin-1 Is Essential for Neural Crest and Cranial Placodes Formation in Xenopus. Biochemical and Biophysical Research Communications, 495, 2257-2263. https://doi.org/10.1016/j.bbrc.2017.12.127

[16] St. Johnston, D. and Nüsslein-Volhard, C. (1992) The Origin of Pattern and Polarity in the Drosophila Embryo. Cell, 68, 201-219. https://doi.org/10.1016/0092-8674(92)90466-P

[17] Anderson, K.V. (1998) Pinning Down Positional Information: Dorsal-Ventral Polarity in the Drosophila Embryo. Cell, 95, 439-442. https://doi.org/10.1016/S0092-8674(00)81610-4

[18] Kronia, L., Whitefield, Z.J., Yuan, B., Dzeyk, K., Kirkpatrick, J., Krijgsreld, J. and Orr-Weaver, T.L. (2014) Quantitative Proteomics Reveals the Dynamics of Protein Changes during Drosophila Oocyte Maturation and Oocyte-to-Embryo Transition. Proceedings of the National Academy of Sciences of the United States of America, 111, 16023-16928. https://doi.org/10.1073/pnas.1418657111

[19] Tarkowski, A.K. (1959) Experiments on the Development of Isolated Blastomeres of Mouse Eggs. Nature, 184, 1286-1287. https://doi.org/10.1038/1841286a0

[20] McLaren, A. (1976) Mammalian Chimeras. Cambridge University Press, Cambridge, UK.

[21] Kelly, S.J. (1977) Studies of the Developmental Potential of 4- and 8-Cell Stage Mouse Blastomeres. Journal of Experimental Zoology, 200, 365-376. https://doi.org/10.1002/jez.1402000307

[22] Gardner, R.L. (1985) Clonal Analysis of Early Mammalian Development. Philosophical Transactions of the Royal Society, 312, 163-178. https://doi.org/10.1098/rstb.1985.0186

[23] Papaioannou, V.E., Gardner, R.L., McBurney, M.W., Babinet, C. and Evans, M.J. (1978) Participation of Cultured Tetratocarcinoma Cells in Mouse Embryogenesis. Journal of Embryology and Experimental Morphology, 44, 93-104.

[24] Illmensee, K. and Stevens, L.C. (1979) Tetramas and Chimeras. Scientific American, 240, 120-132. https://doi.org/10.1038/scientificamerican0479-120

[25] Robertson, E.J. (1986) Pluripotential Stem Cell Line as a Route into the Mouse Germ Line. Trends in Genetics, 2, 9-13. https://doi.org/10.1016/0168-9525(86)90161-7

[26] Williams, R.L., Hilton, D.J., Pease, S., Wilson, T.A., Stewart, C.L., Gearing, D.P., Wagner, E.F., Metcalf, D., Nicola, N.A. and Gough, N.M. (1988) Myaloid Leukemia Inhibitory Factor Maintains the Developmental Potential of Embryonic Stem Cells. Nature, 336, 685-687. https://doi.org/10.1038/336684a0

[27] Capecchi, M.R. (1989) The New Mouse Genetics: Altering the Genome by Gene Targeting. Trends in Genetics, 5, 70-76. 
https://doi.org/10.1016/0168-9525(89)90029-2

[28] The C. elegans Genome Sequencing Consortium (1998) Genome Sequence of the nematoda $C$. elegans. A Platform for Investigating Biology. Science, 282, 2012-2018. https://doi.org/10.1126/science.282.5396.2012

[29] Adams, M.D., Ceiniker, S.E., Holt, R.A., Evans, C.A., Gocayne, J.D., Scherer, S.E., Li, P.N., Hoskins, R.A., Galle, R.F., George, R.A. and Lewis, S.E. (2000) The Genome Sequence of Drosophila melanogaster. Science, 287, 2185-2195. https://doi.org/10.1126/science.287.5461.2185

[30] The Arabidopsis Genome Initiative (2001) Analysis of the Genome Sequence of the Flowering Plant Arabidopsis thaliana. Nature, 408,796-815. https://doi.org/10.1038/35048692

[31] Venter, J.C., Adams, M.D., Myers, E.W., Li, P.W., Mural, R.J., Sutton, G.G., Smith, H.O., Yandell, M., Evans, C.A., Holt, R.A., Gocayne, J.D., et al. (2001) The Sequence of the Human Genome. Science, 291, 1304-1351. https://doi.org/10.1126/science.1058040

[32] Alberts, B., Bray, D., Lewis, J., Raff, M., Roberts, K. and Watson, J.D. (1994) Molecular Biology of the Cell. 3rd Edition, Garland Publishing Inc., New York, 721-943.

[33] Hart, G.W. (1997) Dynamic O-Linked Glycosylation of Nuclear and Cytoskeletal Proteins. Annual Review of Biochemistry, 66, 315-335. https://doi.org/10.1146/annurev.biochem.66.1.315

[34] Baroni, M.D., Monti, P. and Alberghina, L. (1994) Represion of Growth-Regulated G1 Cyclin Expression by Cyclic AMP in Budding Yeast. Nature, 371, 339-342. https://doi.org/10.1038/371339a0

[35] Tokiwa, G., Tyers, M., Volpe, T. and Futcher, B. (1994) Inhibition of G1 Cyclin Activity by the Ras/cAMP Pathway in Yeast. Nature, 371, 342-345. https://doi.org/10.1038/371342a0

[36] Ising, E. (1925) Beitrag zur theorie des ferromagnetismus. Zeitschrift für Physik, 31, 253. https://doi.org/10.1007/BF02980577

[37] van Kampen, N.G. (1962) Fundamental Problems in Statistical Mechanics. Elsevier, Amsterdam, 173.

[38] Kubo, R., Matsuo, K. and Kitahara, K. (1973) Fluctuation and Relaxation of Macrovariables. Journal of Statistical Physics, 9, 51-96. https://doi.org/10.1007/BF01016797

[39] Takahashi, K. and Yamanaka, S. (2006) Induction of Pluripotent Stem Cells from Mouse Embryonic and Adult Fibroblast Cultures by Defined Factors. Cell, 126, 663-676. https://doi.org/10.1016/j.cell.2006.07.024

[40] Tanabe, K., Nakamura, M., Narita, M., Takahashi, K. and Yamanaka, S. (2013) Maturation, Not Initiation, Is the Major Roadblock during Reprogramming to Ward Pluripotency from Human Fibroblasts. Proceedings of the National Academy of Sciences of the United States of America, 110, 12172-12179. https://doi.org/10.1073/pnas.1310291110 